HIAS-E-50

\title{
One million miles to go: taking the axiomatic road to defining exploitation
}

\author{
Roberto Veneziani \\ School of Economics and Finance, Queen Mary University of London, Mile End Road, London E1 4NS, UK \\ Naoki Yoshihara \\ The Institute of Economic Research, Hitotsubashi University, Naka 2-1, Kuni- tachi, Tokyo 186-8603, Japan
}

October 2015

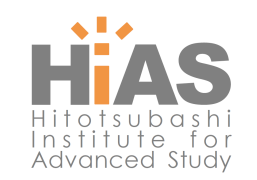

Hitotsubashi Institute for Advanced Study, Hitotsubashi University 2-1, Naka, Kunitachi, Tokyo 186-8601, Japan

tel:+81 425808604 http://hias.ad.hit-u.ac.jp/

HIAS discussion papers can be downloaded without charge from: http://hdl.handle.net/10086/27202

https://ideas.repec.org/s/hit/hiasdp.html

All rights reserved. 


\title{
One million miles to go: taking the axiomatic road to defining exploitation*
}

\author{
Roberto Veneziani ${ }^{\dagger} \quad$ Naoki Yoshihara ${ }^{\ddagger}$
}

October 29, 2015

\begin{abstract}
This paper analyses the Marxian theory of exploitation. The axiomatic approach standard in social choice theory is adopted in order to study the concept of exploitation - what it is and how it should be captured empirically. Two properties are presented that capture some fundamental Marxian insights. It is shown that, contrary to the received view, there exists a nonempty class of definitions of exploitation that preserve the relation between exploitation and profits - called Profit-Exploitation Correspondence Principle - in general economies with heterogeneous agents, complex class structures, and production technologies with heterogeneous labour inputs. However, among the main approaches, only the so-called 'New Interpretation' satisfies the Profit-Exploitation Correspondence Principle in general.
\end{abstract}

JEL: B51.

Keywords: Exploitation, profits, axiomatic analysis.

*We thank Gary Dymski, Don Katzner, Gil Skillman, Peter Skott, two anonymous referees, the editors of this journal, and participants in the Analytical Political Economy workshop and the American Economic Association conference (Philadelphia) for useful comments and suggestions. We worked on this paper while visiting the University of Massachusetts, Amherst: their support and hospitality is gratefully acknowledged. The usual disclaimer applies.

$\dagger$ (Corresponding author) School of Economics and Finance, Queen Mary University of London, Mile End Road, London E1 4NS, United Kingdom (r.veneziani@qmul.ac.uk)

$\ddagger$ The Institute of Economic Research, Hitotsubashi University, Naka 2-1, Kunitachi, Tokyo 186-0004, Japan (yosihara@ier.hit-u.ac.jp) 


\section{Introduction}

After a wave of intense research and debates in the 1970s and 1980s, the Marxian theory of exploitation has moved to the sidelines of academic economics. ${ }^{1}$ The notion of exploitation has never had much appeal for mainstream economists. But heterodox scholars, in the main, also seem sceptical about the possibility of developing a logically coherent and empirically meaningful concept of exploitation. Two broad (and related) sets of objections have been moved against the Marxian theory of exploitation.

First, it is unclear what Marxian exploitation actually is. At a general level, it can be defined as the (forced) extraction of surplus labour, or as a power relation leading to systematic differences between the labour contributed (in some relevant sense) by workers in productive activities and the labour that they receive (in some relevant sense) in return. As intuitive as these general formulations are, outside of stylised two-class economies with linear technologies and homogeneous labour, the notions of surplus labour, or of labour contributed and received, have no obvious interpretation. Different definitions of exploitation can be, and have in fact been proposed that rely on different interpretations of these concepts (e.g., Okishio [33]; Morishima [31]; Roemer [37]; Flaschel [8]; Duménil [5]; Foley [10]). Actually, as Steedman [43] famously argued, it is not even clear that these concepts - and, a fortiori, Marxian exploitation - can be coherently defined.

Second, it is unclear what the concept of exploitation does. The conceptual problems, anomalies and counterexamples identified in the literature have led to increasingly complex and often counterfactual definitions that have progressively lost intuitive appeal, and also their applicability to actual capitalist economies, calling into question the usefulness of the notion of exploitation. At a methodological level, this is often attributed to the mathematical turn taken by the debate on exploitation in the 1970s, which, according to critics, has shifted the focus from the broader conceptual issues to relatively minor technical details.

This paper defends the relevance of Marxian exploitation in general production economies with heterogeneous labour, while adopting a formal ap-

\footnotetext{
${ }^{1}$ To be sure, research on exploitation theory has not stopped. See, for example, recent debates on financial exploitation and expropriation (Lapavitsas [18, 19]; Fine [7]) and on the so-called Temporal Single-System Interpretation of value theory (Freeman and Carchedi [13]; Mohun [29]; Veneziani [46]). Nonetheless, these debates have had a relatively limited impact outside the confines of Marxist economics.
} 
proach. First, a definition is proposed, which is conceptually related to the 'New Interpretation' (Duménil [5]; Foley [10]; Duménil et al [6]). According to this definition, an agent is exploited (resp., an exploiter) if and only if the labour she contributes is greater (resp., lower) than the share of aggregate social labour that she receives via her income. This approach defines exploitation as a feature of the competitive allocation of social labour rather than as the result of productive inefficiencies, or labour market imperfections. Unlike the received definitions, it has a clear empirical content, for it is firmly anchored to the actual data of the economy. Further, we show that it preserves some widely shared positive and normative intuitions concerning the notion of exploitation, including the existence of a robust relation between exploitation and profits, in economies with heterogeneous labour. This is an important and surprising result.

At a general, theoretical level, it is often argued that the concept of exploitation is central in Marxian economics because it links the visible, epiphenomenal characteristics of capitalist economies (as revealed by monetary variables) with deeper, structural forces and mechanisms. Whether profits are indissolubly linked to exploitation is important, in this perspective, both if exploitation is meant to uncover the fundamental forces underlying the dynamics of accumulation in capitalist economies; and if it is an essentially normative criterion to evaluate, and indict, capitalism. For, given private ownership of productive assets, one should expect profits to be a counterpart of the transfer of social surplus and social labour from workers to capitalists, and one of the causes of inequalities in well-being freedom.

The existence of a relation between exploitation and profits has been famously proved by Okishio [34] in linear economies with homogeneous labour. Given its relevance the result has been dubbed the Fundamental Marxian Theorem (henceforth, FMT), and has sparked a substantial literature exploring its generality and relevance. ${ }^{2}$ While retaining the assumption of homogeneous labour, Steedman [43] famously proved that, under Okishio's standard definition, the FMT does not hold in von Neumann economies with joint production. Morishima [31] and Roemer [37] proposed two alternative definitions of exploitation that meet Steedman's critique. Nonetheless, these results are far from conclusive: Yoshihara and Veneziani [53] have shown that

\footnotetext{
${ }^{2}$ The literature is too vast for a comprehensive list of references. In addition to the classic contributions cited in the text, it is worth mentioning the more recent discussions by Mohun [29]; Flaschel [8]; Yoshihara and Veneziani [53].
} 
neither Morishima's [31] nor Roemer's [37] definition preserves the FMT in economies with a convex technology and homogeneous labour. Perhaps more importantly, the robustness of the FMT in more general economies with heterogeneous skills and labour inputs is an open question. ${ }^{3}$ Indeed, it is often argued that heterogeneous labour poses intractable problems for Marxian exploitation theory.

As explained in section 4 below, our analysis bears only a broad conceptual relation with the FMT literature. Yet, in the light of the debates on the FMT, it is remarkable that, if the New Interpretation is adopted, then it is possible to establish a robust relation between exploitation and profits in general economic environments with heterogeneous labour. Indeed, the New Interpretation is the only definition - among the main ones in the literature with this property. Veneziani and Yoshihara [49] established this conclusion in economies with homogeneous labour inputs, and focusing on a specific set of equilibrium allocations. Here we significantly generalise their analysis by focusing on a larger set of economies and allocations, and tackling the conceptually fundamental and formally more complex case of heterogeneous labour. ${ }^{4}$

The second contribution of the paper is methodological. An axiomatic approach is developed, whereby the desirable characteristics of a definition of exploitation are stated formally. The axiomatic approach is standard in several fields in economics, most notably in social choice theory. Yet, as this is one of its first applications to Marxian and classical themes, ${ }^{5}$ the next subsection addresses some methodological issues related to the use of mathematics in economics. The aim is not to provide a general methodological discussion, but rather to explain why the axiomatic approach adopted in this paper is both appropriate and insightful in the analysis of the concept of exploitation. The uninterested reader may safely skip to section 2 .

\footnotetext{
${ }^{3}$ The FMT has been extended to consider heterogeneous labour (e.g., Fujimori [14]; Krause [16]; Bowles and Gintis [2]), but only in the context of standard linear economies.

${ }^{4}$ The axioms and definitions in Veneziani and Yoshihara [49] can be obtained as a special case of those presented here, if one assumes homogeneous labour and focuses on equilibrium allocations.

${ }^{5}$ Notable exceptions include Yoshihara [51]; Veneziani and Yoshihara [49]; Flaschel et al. [9].
} 


\subsection{Taking the axiomatic road}

This paper addresses the issue of the appropriate definition of exploitation and measure of exploitative relations in capitalist economies. The motivation of our analysis is not the lack, but the wealth of plausible candidates: as noted above, many different definitions can be, and have in fact been proposed, which incorporate different positive and normative intuitions.

The fundamental question is how to choose among all of the existing and the conceivable definitions. Thus far, the debate has largely been reactive: new definitions have often emerged as the product of a process of adjustment of the theory to various anomalies and counterexamples identified in the literature. We adopt a different approach. Rather than proposing another definition, and comparing it with the existing alternatives, we develop an axiomatic framework to analyse what exploitation is, and how it should be measured. The axiomatic method is used to rigorously and explicitly state the normative and positive foundations of the notion of exploitation. ${ }^{6}$

The starting point of our analysis is that the concept of exploitation has a quantitative dimension. This is not to say that exploitation can or should be reduced to a quantitative phenomenon. Purely distributive approaches that reduce exploitation to an inequality in productive assets (such as Roemer's [37] property relations approach), for example, are ultimately unsatisfactory. For exploitative social relations arguably involve some form of power, force, or coercion, which need not be clearly measurable (Veneziani $[47,48]$ ).

Yet the concept of exploitation also has an inherently quantitative dimension, such that it is meaningful to say that "economy A has become significantly more exploitative over the past four decades", or that "exploitation is worse in country B than in country C". Exploitation diagnoses the process

\footnotetext{
${ }^{6} \mathrm{~A}$ point of language should be clarified here. The term 'axiomatic' is sometimes used to define any formal, deductive reasoning starting from given premises (axioms). From this perspective, all mathematical Marxian economics is 'axiomatic.' We use the term axiomatic in a narrower sense to denote the method that is standard in social choice theory and inequality analysis (and therefore a strict subset of formal techniques). In his analysis of the 'axiomatic method' in social choice theory, Thomson ([45], p.332) puts it thus: "An axiomatic study has the following components: 1. It begins with the specification of a domain of problems, and the formulation of a list of desirable properties of solutions for the domain. 2. It ends with ... descriptions of the families of solutions satisfying various combinations of the properties." The earlier literature on Marxian exploitation theory (formal or otherwise) was not axiomatic in this sense, as it moved from the specification of a domain of problems (economies) directly to the proposal of a given solution (definition of exploitation).
} 
through which "certain inequalities in incomes are generated by inequalities in rights and powers over productive resources: the inequalities occur, in part at least, through the ways in which the exploiters, by virtue of their exclusionary rights and powers over resources, are able to appropriate labour effort of the exploited" (Wright [50], p.1563). This paper focuses precisely on the quantitative dimension of exploitation and on the most appropriate way of capturing this aspect of exploitative social relations.

It may be objected that the possibility of measuring a certain phenomenon does not imply that formal tools should be used to study it. ${ }^{7}$ Critical realism, for example, has long argued against the mainstream insistence that mathematical methods be used always and everywhere in economics. According to critical realists, social reality is most plausibly construed as an open, structured, dynamic and internally related system and mathematicaldeductivist methods are inappropriate in the causal-explanatory analysis of open systems (Lawson [22, 24]).

A thorough discussion of the role of formal tools in social theorising goes beyond the scope of this paper. But it is worth explaining why the main objections against the use of mathematics in economics do not apply here.

This paper examines the category of "exploitation", in order to understand what it means, and how it can be captured empirically. Our theoretical effort can be conceived of as philosophical underlabouring a central concept of Marxian economics and we use the axiomatic method typical of social choice theory in order to identify the desirable properties of a definition (and measure) of exploitation. The analysis is not based on any assumptions whether explicit or implicit - about the nature of social reality as a closed system, or about the pervasiveness of strict regularities and constant conjunctions of events. ${ }^{8}$ Neither closures of causal sequence - where some event $y$ is causally conditioned by some other event(s) $x$ (Lawson [22], p.15) - nor the weaker closures of concomitance - "where the events $[x$ and $y]$ are correlated, but where neither causally conditions the other" (ibid.) - underlie the use of formal tools in our analysis. ${ }^{9}$ As we are trying to uncover neither

\footnotetext{
${ }^{7}$ Conversely, however, the fact that some variables are not measurable does not rule out a priori the use of formal tools (Katzner [15]).

${ }^{8} \mathrm{It}$ is worth stressing that ours are not general methodological claims about the intrinsic properties of the axiomatic method (as a subset of the set of possible mathematical techniques) in the analysis of social reality. Rather, the point is that the axiomatic approach is appropriate in this specific context, given the nature of the problem investigated.

${ }^{9}$ If one stipulates a property of a definition (and measure) of exploitation whereby,
} 
causal mechanisms nor correlations of events, our formal analysis does not presuppose the existence of either type of closure. ${ }^{10}$

Ours is an investigation in scientific ontology and in this respect it is similar in nature to Sen's [39] analysis of the concepts of functionings and capabilities (see Martins $[25,26]$ ). ${ }^{11}$ Because of the nature of our inquiry, the axiomatic framework does not incorporate any hypotheses concerning causal laws (or even tendencies) within a predictionist perspective (Lawson [22], p.60). Rather, it aims to clarify the social category of exploitation and to capture exploitative relations a posteriori, focusing on the state of the economy at a given point in time. It is therefore conceptually analogous to the approach used to identify appropriate measures of poverty and inequality (Foster [12]), or labour productivity (Flaschel et al [9]).

The axioms are thus abstract in that they incorporate relevant philosophical views about the nature and the positive and normative foundations of Marxian exploitation. But they are also empirically oriented, in that they focus on observable magnitudes, and do not rely on "claims that are believed to be false of our world, and of any really possible counterfactual world" (Lawson [23], p.766). Indeed, the key condition for the relevance of our axiomatic analysis is that the main variables (prices, wages, output, labour time, and so on) be conceptually well-defined, meaningful, and empirically measurable within the appropriate temporal and spatial context. ${ }^{12}$

The axiomatic framework also precisely identifies the domain and scope of the analysis by focusing on a set of economic agents and on a class of economic

exploitation $(y)$ is said to exist whenever aggregate profits are positive $(x)$, then this is neither a claim about profits 'causing' exploitation, nor a claim about the existence of an empirical regularity whereby $x$ and $y$ occur together. It is a definitional claim stating that if we observe $x$ then an appropriate measure of exploitation should yield $y$.

${ }^{10}$ Nor does it presuppose the existence of "conditional closures" (Setterfield [41]), "quasiclosures" (Downward et al. [4]) or "local closures" (Lawson [21]).

11 "[A]n approach which is centred on the characterisation of objects for ethical valuation, such as 'functionings' and 'capabilities', is essentially an ontological approach, which provides the objects that we may then use when engaging in democratic ethical valuation." (Martins [26], p.150).

${ }^{12}$ The same condition is necessary for the use of descriptive statistics in empirical analyses advocated by Lawson ([20], p.221), or for the adoption of econometric techniques within a triangulation strategy consistent with a critical realist outlook (see, for example, Part II of Downward [3]). As Lawson ([20], p.69) puts it, "the determination of summary statistics of a body of data such as means or growth rates of some phenomenon can be included under the rubric of econometrics. Such activities are not being questioned here ... . The specific realist emphasis that I am pursuing is not an anti-empirical one." 
environments and allocations. But this by no means entails a reductionist perspective. On the one hand, no strong restrictions are imposed on agent behaviour or on the institutional mechanisms (market-based or otherwise) regulating economic interactions. The axioms incorporate no assumptions concerning individuals' ontology (including their selfish or ethical motivations, the origin of their preferences, or the notion of individual rationality), the nature of social interactions, and so on. On the other hand, although the axioms focus on the exploitation status of individuals at a given point in time, they do not entail a commitment to atomism, to a static view of social reality, or to the existence of a fixed, given unit of analysis. Depending on the object of analysis, the axiomatic system can be modified to incorporate aggregate properties relating, for example, to the exploitation status of social classes, or to the overall level of exploitation in the economy, and without reference to individuals; or relational properties incorporating the idea that exploitation is a social relation such that an agent is exploited if and only if there is someone exploiting her. But one can also introduce $d y$ namic properties focusing, for example, on the relation between exploitation and accumulation, or specifically concerning the measurement of exploitation when agents save; or distinguishing between the exploitation status of individuals within each period and over the course of their entire lives.

To be sure, the intuitive appeal of a definition is fundamental, and a certain definition should provide the right answers in situations in which we feel that intuition is a reliable guide. The axiomatic method is not "a substitute for intuition ... but instead ... a way of articulating [the intuitions that hold in specific situations] into operationally useful conditions pertaining to an entire class of cases" (Thomson [45], p.356). Indeed, as shown by the often surprising impossibility results obtained in social choice theory, or by the very difficulty in providing a definition of exploitation that preserves key Marxian insights, intuition alone can be insufficient. When delineating the properties of the appropriate definition of exploitation, "Informal insights, important as they are, cannot replace the formal investigations that are needed to examine the congruity and cogency of combinations of values and of apparently plausible demands" (Sen [40], p.353).

Of course, the axiomatic method does not necessarily lead to univocal conclusions: one can reject any of the axioms below, and propose a new one, possibly leading to an alternative definition of exploitation. This indeterminacy is not a property of axiomatic analysis per se: it is inherent in all social theorising and it simply reflects different positive and normative in- 
tuitions. ${ }^{13}$ If anything, the axiomatic method has the advantage of making such intuitions explicit, thus forcefully directing research and debate to the foundational issues concerning the nature and measurement of exploitation.

This is an important point. Unlike in much of the mainstream, in this paper the axiomatic method is not used in a purely instrumental way, for example in order to generate predictions that match empirical data, and regardless of the actual relevance of the axioms (Lawson [22, 24]). The content of the axioms is central to our analysis, and the relevance and meaningfulness of the conclusions ultimately depends on it. For, "The relevance of an axiomatic result depends entirely on the acceptability or usefulness of its constituent properties" (Foster [12], p.367).

\section{Economic states}

The aim is to analyse exploitation without imposing significant restrictions on agents' behaviour, market structure, and so on. Therefore we keep the description of the economic environment to a bare minimum. ${ }^{14}$ Consider an economy with $N$ agents, $n$ commodities, and $T$ types of labour. Let $\mathcal{N}$ be the set of agents with generic element $\nu$ and let $\mathcal{T}$ be the set of types of labour with generic element $\tau$.

Technology is described by a production set $P$ with elements - activities - of the form $\alpha=\left(-\alpha_{l},-\underline{\alpha}, \bar{\alpha}\right)$ where $\alpha_{l} \equiv\left(\alpha_{l \tau}\right)_{\tau \in \mathcal{T}}$ is the (nonnegative) profile of the $T$ labour inputs used in production, measured in hours $;{ }^{15} \underline{\alpha}$ is the (nonnegative) profile of the $n$ inputs of produced goods; and $\bar{\alpha}$ is the (nonnegative) profile of the outputs of the $n$ goods. ${ }^{16}$ By measuring labour inputs in terms of time, this description of the technology includes standard economies as a special case.

For example, suppose that the production of one unit of each good requires a fixed amount of the $n$ commodities and of the $T$ types of labour. As

\footnotetext{
${ }^{13}$ As Sen ([38], p.187) noted in his discussion of the labour theory of value, "it is not really surprising that different conventions for calculating aggregate labour magnitudes would exist and also appear natural in different contexts. The source of these ambiguities ... [rests] in the basic multiplicity of motivation underlying the labour theory."

${ }^{14}$ The model and notation are a generalisation of Roemer's [36] classic framework.

${ }^{15}$ Throughout the paper, all variables and vectors are assumed to belong to a Euclidean space $\mathbb{R}^{k}$ of appropriate dimensionality $k$.

${ }^{16}$ All variables should be dated. However, because we analyse exploitative relations at a given point in time, we drop the time subscript for notational convenience.
} 
in standard input-output theory, production techniques can be represented by a pair $(\boldsymbol{A}, \boldsymbol{L})$, where $\boldsymbol{A}$ is a $n \times n$ non-negative (productive and indecomposable) Leontief matrix of material coefficients and $\boldsymbol{L} \equiv\left[L^{1}, \ldots, L^{n}\right]$ is a $T \times n$ non-negative matrix of direct labour coefficients (with at least one strictly positive element in each row and column), where $L^{i}$ is a vector describing the amount of each type of labour necessary to produce one unit of good $i$. Then, $P \equiv\left\{\alpha=\left(-\alpha_{l},-\underline{\alpha}, \bar{\alpha}\right) \mid \exists x \geqq \mathbf{0}:(-\boldsymbol{L} x,-\boldsymbol{A} x, x)=\alpha\right\}$ and we say that $P$ is representable by a Leontief production technique $(\boldsymbol{A}, \boldsymbol{L})$.

The net output vector arising from $\alpha$ is denoted as $\widehat{\alpha} \equiv \bar{\alpha}-\underline{\alpha}$ and the set of efficient activities in $P$ is denoted as $\partial P .{ }^{17}$ In the rest of the paper, we assume that: technology displays constant returns to scale; firms can decide not to activate any process; and the production of any output requires some labour and some capital. ${ }^{18}$ These restrictions are rather mild and standard in heterodox (and even mainstream) approaches.

Each agent $\nu$ is endowed with a (nonnegative) vector of $n$ productive assets, $\omega^{\nu}$, and a nonempty set of types of labour $\mathcal{T}^{\nu} \subseteq \mathcal{T}$ that can be used in production. The total amount of time that each $\nu$ can use either productively (possibly in different types of labour) or in leisure activities, is normalised to one. Let $C$ be the set of all conceivable choices of each agent with generic element $\left(c^{\nu}, \lambda^{\nu}\right)$, where $c^{\nu}$ is a nonnegative vector describing $\nu$ 's consumption of the $n$ goods and $\lambda^{\nu}=\left(\lambda_{1}^{\nu}, \ldots, \lambda_{T}^{\nu}\right)$ describes the amount of time of each type of labour spent by $\nu$ in productive activities, where $\lambda_{\tau}^{\nu} \in[0,1]$ for all types of labour $\tau \in \mathcal{T}^{\nu}$ that $\nu$ is endowed with; $\lambda_{\tau}^{\nu}=0$ for all other labour types $\tau \in \mathcal{T} \backslash \mathcal{T}^{\nu}$; and $\sum_{\tau \in \mathcal{T}^{\nu}} \lambda_{\tau}^{\nu} \leqq 1$.

Let $(p, w)$ be the (row) vector describing the (positive) prices of the $n$ commodities and the (nonnegative) wages of the $T$ types of labour. Let $\pi^{\max }$ be the maximum profit rate that can be obtained from production activities at prices $(p, w)$, and let $P^{\pi}(p, w)$ be the set of production processes that yield the maximum profit rate at $(p, w) \cdot{ }^{19}$

Our analysis does not depend on any specific assumptions on individual behaviour, or on the institutional framework in which agents interact. However, both the key axioms and the main results apply only to a class of economic scenarios which may, or may not, turn out to be true ex post. Yet they are sufficiently general and theoretically relevant to warrant investiga-

\footnotetext{
${ }^{17}$ Formally: $\partial P \equiv\left\{\alpha \in P \mid \nexists \alpha^{\prime} \in P\right.$ such that $\alpha_{i}^{\prime}>\alpha_{i}$ for all $\left.i\right\}$.

${ }^{18}$ These assumptions on $P$ are stated formally in appendix A.1.

${ }^{19}$ Formally, $\pi^{\max }=\max _{\alpha \in P} \frac{p \widehat{\alpha}-w \alpha_{l}}{p \underline{\alpha}}$ and $P^{\pi}(p, w)=\left\{\alpha \in P \mid \pi^{\max }=\frac{p \widehat{\alpha}-w \alpha_{l}}{p \underline{\alpha}}\right\}$.
} 
tion and to cover a large set of possible cases.

An economic environment is a set of agents, $\mathcal{N}$; a production set, $P$; a consumption space, $C$; a profile of agents' labour endowments, $\left(\mathcal{T}^{\nu}\right)_{\nu \in \mathcal{N}}$; and a profile of agents' endowments of productive assets, $\left(\omega^{\nu}\right)_{\nu \in \mathcal{N}}$. Given an economic environment, an economic outcome is a price vector, $(p, w)$, a profile of consumption and labour decisions $\left(c^{\nu}, \lambda^{\nu}\right)_{\nu \in \mathcal{N}}$, and a profile of production activities operated by agents $\left(\alpha^{\nu}\right)_{\nu \in \mathcal{N}}$, with aggregate production activity, $\alpha^{p, w}=\sum_{\nu \in \mathcal{N}} \alpha^{\nu}$, such that: ${ }^{20}$ (i) aggregate net output can at least provide for consumption, $\widehat{\alpha}^{p, w} \geqq \sum_{\nu \in \mathcal{N}} c^{\nu}$; (ii) aggregate profits are nonnegative, $p \widehat{\alpha}^{p, w}-w \alpha_{l}^{p, w} \geqq 0 ;$ (iii) aggregate production maximises the profit rate, $\alpha^{p, w} \in P^{\pi}(p, w)$ with $\alpha_{l}^{p, w}=\sum_{\nu \in \mathcal{N}} \lambda^{\nu}$; and (iv) individual expenditure does not exceed individual (capital and wage) income, $p c^{\nu}=\pi^{\max } p \underline{\alpha}^{\nu}+w \lambda^{\nu}$ for each agent $\nu$. A pair of an economic environment and an economic outcome is an economic state (henceforth, ES).

The concept of $E S$ is very general, with no substantive restrictions on behaviour, technology or institutions. Conditions (i) and (ii) are hardly demanding. Condition (iii) is only slightly more restrictive in that it postulates that producers activate profit-rate-maximising processes. This is consistent with Marx's ([28], ch.10) analysis of capitalist behaviour and is common in heterodox approaches (including Sraffian price theory). It is also theoretically justified because it is desirable to obtain a definition that can capture exploitation under optimal capitalist behaviour. In Marxian theory, exploitation is the product of capitalist social relations, rather than mistakes, technical inefficiencies, or market imperfections. ${ }^{21}$ Condition (iv) postulates that individual expenditure is subject to the budget constraint. This yields no significant loss of generality and it does not rule out savings, as the bundle $c^{\nu}$ is not necessarily restricted to consumption goods and services. Further, it is theoretically justified because Marxian exploitation is the product of capitalist social relations, and in particular of the wage relation, rather than credit markets and individual life-cycle decisions.

The notion of ES does not incorporate any of the standard features of mainstream models such as utility functions, selfish optimising (or even boundedly rational) consumers, and differentiable production functions. The

\footnotetext{
${ }^{20}$ For all vectors $x, y \in \mathbb{R}^{n}, x \geqq y$ if and only if $x_{i} \geqq y_{i}(i=1, \ldots, n) ; x \geq y$ if and only if $x \geqq y$ and $x \neq y ; x>y$ if and only if $x_{i}>y_{i}(i=1, \ldots, n)$.

${ }^{21}$ The equality $\alpha_{l}^{p, w}=\sum_{\nu \in \mathcal{N}} \lambda^{\nu}$ does not imply any form of labour market equilibrium or optimising behaviour. It simply states that the labour used in production is performed by the agents in the economy.
} 
production set is sufficiently general to allow for the existence of a differentiable production function, but it does not postulate it and nothing depends on neoclassical assumptions of any sort. Nor does the definition of $E S$ rely on the concept of equilibrium. According to Lawson [24], a focus on equilibrium is a requirement of mathematical models proceeding in deductive mode to specify causal influences, but our inquiry is of a different, ontological nature.

Perhaps more importantly, the concept of $E S$ is not a description of the functioning of the economy: how it is structured, what agents do or how they choose, and so on. Rather, it acts formally as a domain condition: it defines the scope and boundaries of the analysis. Social scientific theorising is always context-specific, and the definition of ES makes the context explicit. It is important to note, however, that such boundaries are rather wide indeed (and significantly wider than in the literature), for conditions (i)-(iv) can obtain in a range of economies and under many different assumptions concerning, e.g., institutions and behaviour. ${ }^{22}$ Further, although it is an open question whether our conclusions hold even more generally, the key insights of the paper are robust to several changes in the definition of $E S{ }^{23}$

\section{Defining Marxian exploitation}

In Marxian theory, exploitation can be seen in two related but slightly different ways. One can focus on the difference between the labour performed by workers and the labour socially necessary to produce the goods they consume. Or, one can conceptualise exploitative social relations as characterised by systematic differences between the labour that agents 'contribute' to the economy and the labour they 'receive'. As soon as one moves away from the simplest linear economies, none of these concepts is clearly defined and, as already noted, various definitions have been proposed which reflect different views concerning the concept of exploitation.

Most (though not all) of the previous debates have revolved around the appropriate definition of socially necessary labour time, or of the labour received by agents. In order to focus on this issue, contributors have analysed

\footnotetext{
${ }^{22}$ The set of economic states is nonempty. It contains, for example, generalisations of Roemer's [36, 37] economies as special cases (see the Addendum).

${ }^{23}$ For example, all results continue to hold if condition (i) is weakened to require that aggregate net output is at least as high as the consumption of any individual agent $\left(\widehat{\alpha}^{p, w} \geqq\right.$ $c^{\nu}$ for each $\nu$ ), or, indeed, that it is strictly positive.
} 
economies with homogeneous labour, in which the labour performed by workers, or the labour that they contribute to the economy is uncontroversial: it corresponds to the labour time spent in productive activities. In more general economies, however, these concepts are not trivial.

In this section, we extend some of the main definitions to economies with heterogeneous labour. The key step is to acknowledge that although the notion of labour performed, or contributed is theoretically important, the differences between alternative approaches lie elsewhere. Therefore we adopt a common definition of the labour performed, or contributed as the value of labour time spent in production. Formally, at any ES, the amount of labour contributed by agent $\nu$ who supplies $\lambda^{\nu}$ is equal to $w \lambda^{\nu}{ }^{24}$

This approach is consistent with the classical economists' view on how to convert different types of labour into a single unit, whereby "the different kinds of labour are to be aggregated via the (gold) money wage rates" (Kurz and Salvadori [17], p.324). According to Smith, for example,

"It is often difficult to ascertain the proportion between two different quantities of labour. The time spent in two different sorts of work will not always alone determine this proportion. The different degrees of hardship endured, and of ingenuity exercised, must likewise be taken into account. There may be more labour in an hour's hard work, than in two hours easy business; or in an hour's application to a trade which it cost ten years labour to learn, than in a month's industry, at an ordinary and obvious employment. But it is not easy to find any accurate measure either of hardship or ingenuity. In exchanging, indeed, the different productions of different sorts of labour for one another, some allowance is commonly made for both. It is adjusted, however, not by any accurate measure, but by the higgling and bargaining of the market" (Smith [42], ch.V, pp.34-35).

One can similarly interpret Ricardo's ([35], ch.I, section II, p.11) arguments that "The estimation in which different quantities of labour are held, comes soon to be adjusted in the market with sufficient precision ... ,

\footnotetext{
${ }^{24}$ It is worth stressing that $w$ can be interpreted either as the vector of nominal wages, or as expressing the ratios of the wages of each type of labour relative to some benchmark (for example, the wage of simple labour or the average wage). All of our definitions, axioms, and conclusions remain unchanged under either interpretation.
} 
and depend much on the comparative skill of the labourer, and intensity of the labour performed". Despite some debates on the concept of "abstract labour", our approach is also consistent with Marx's ([27], pp.51-2) views on the conversion of complex labour into simple labour. ${ }^{25}$

Indeed, although exploitation theorists often do not provide a complete extension of their definitions to economies with heterogeneous labour, in the main they do endorse (albeit sometimes implicitly) the classical economists' view on the use of wages for the reduction of different types of labour to a single unit (Morishima [30], ch.14; Duménil et al. [6]). ${ }^{26}$

Consider first Morishima's [30, 31] classic definition. At any ES, according to Morishima, the amount of labour received by agent $\nu$, who consumes $c^{\nu}$, corresponds to the minimum amount of labour necessary to produce $c^{\nu}$ as net output. Following Roemer [36, 37], we denote this amount as l.v. $\left(c^{\nu} ; w\right)$, that is, the labour value of $c^{\nu}$ at wages $w .^{27}$ Therefore:

Definition 1: Consider any ES. Agent $\nu \in \mathcal{N}$, who supplies $\lambda^{\nu}$ and purchases $c^{\nu}$, is exploited if and only if $w \lambda^{\nu}>$ l.v. $\left(c^{\nu} ; w\right)$ and an exploiter if and only if $w \lambda^{\nu}<$ l.v. $\left(c^{\nu} ; w\right)$.

Definition 1 has some desirable characteristics, according to Morishima ([31], pp.616-618): the notion of exploitation is well-defined because l.v. $(c ; w)$ is unique, well-defined and nonnegative. ${ }^{28}$ Furthermore, although wages are necessary to convert different types of labour into a single unit, exploitation status can be determined prior to and independent of the prices of commodities, as in classical Marxian theory. ${ }^{29}$

According to Roemer [36, 37], however, Definition 1 is conceptually flawed because it identifies exploitation status based on production techniques that may never be used by profit-maximising capitalists. Like Morishima [31], Roemer [37] focuses on the bundle $c^{\nu}$ actually consumed by agents but argues that its labour content is given by the minimum amount of labour necessary to produce it as net output using profit-rate-maximising production processes,

\footnotetext{
${ }^{25}$ It is also worth noting that Yoshihara and Veneziani [55] have proved that in economies with heterogeneous labour the wage-additive measure is the unique measure of labour content that satisfies a small set of theoretically robust and intuitive axioms.

${ }^{26}$ For a different approach see Okishio [32, 34]; Fujimori [14]; Krause [16].

${ }^{27}$ Formally, for any $E S$ and any $c \in \mathbb{R}_{+}^{n}$, l.v. $(c ; w) \equiv \min \left\{w \alpha_{l} \mid \alpha \in P \& \widehat{\alpha} \geqq c\right\}$.

${ }^{28}$ This follows from assumptions $\mathbf{A 0} \sim \mathbf{A 2}$ in Appendix A.1 (see Roemer [36], Proposition 2.1). The same holds for l.v. $(c ; p, w)$ below.

${ }^{29}$ Definition 1 reduces to Morishima's [30, 31] classic definition in economies with homogeneous labour where $\lambda^{\nu}$ is a scalar. The same holds for Definitions 2 and 3 below.
} 
for only the latter are activated by capitalists. Following Roemer [36, 37], we denote this amount as l.v. $\left(c^{\nu} ; p, w\right)$, that is, the labour value of $c^{\nu}$ at prices $p$ and wages $w .^{30}$ Then:

Definition 2: Consider any ES. Agent $\nu \in \mathcal{N}$, who supplies $\lambda^{\nu}$ and purchases $c^{\nu}$, is exploited if and only if $w \lambda^{\nu}>l . v .\left(c^{\nu} ; p, w\right)$ and an exploiter if and only if $w \lambda^{\nu}<$ l.v. $\left(c^{\nu} ; p, w\right)$.

Although they preserve some important Marxian insights, Definitions 1 and 2 have been criticised because exploitation status depends on counterfactual amounts of labour content (Flaschel [8]). For the production activities yielding $l . v .\left(c^{\nu} ; w\right)$ or $l . v .\left(c^{\nu} ; p, w\right)$ may be different from those actually used in the economy. According to critics, this use of counterfactuals is theoretically undesirable and makes exploitation an empirically vacuous notion, since the computation of l.v. $\left(c^{\nu} ; w\right)$ and $l . v \cdot\left(c^{\nu} ; p, w\right)$ requires information that is normally unavailable, including, in Morishima's own words, "information about all the available techniques of production, actually chosen or potentially usable" ([31], p.617, italics added). ${ }^{31}$

An alternative approach has been recently proposed by Yoshihara and Veneziani $[52,51,49]$. Consider any $E S$ with aggregate production activity $\alpha^{p, w}$. For any nonnegative vector $c$, such that $p c \leqq p \widehat{\alpha}^{p, w}$, the labour content of $c$ is equal to $\tau^{c} w \alpha_{l}^{p, w}$, where $\tau^{c} \in[0,1]$ is such that $\tau^{c} p \widehat{\alpha}^{p, w}=p c .^{32}$ Thus, the labour content of aggregate net output, $\widehat{\alpha}^{p, w}$, is equal to the value of total social labour, $w \alpha_{l}^{p, w}$, and the labour contained in any bundle $c$ (whose value does not exceed national income) is equal to the fraction $\tau^{c}$ of social labour necessary to produce a fraction of aggregate net output, $\tau^{c} \widehat{\alpha}^{p, w}$, that has the same value as $c$. We denote this amount as $l . v .\left(c ; p, w, \alpha^{p, w}\right)$, that is, the labour value of $c$ at prices $p$, wages $w$ and aggregate production, $\alpha^{p, w}$. Then:

Definition 3: Consider any ES. Agent $\nu \in \mathcal{N}$, who supplies $\lambda^{\nu}$ and purchases $c^{\nu}$ is exploited if and only if $w \lambda^{\nu}>$ l.v. $\left(c^{\nu} ; p, w, \alpha^{p, w}\right)$ and an exploiter if and only if $w \lambda^{\nu}<\operatorname{l.v} \cdot\left(c^{\nu} ; p, w, \alpha^{p, w}\right)$.

Definition 3 is conceptually related to the 'New Interpretation' of Duménil [5] and Foley [10]. In fact, for any agent $\nu, \tau^{c^{\nu}}$ represents $\nu$ 's share of national

\footnotetext{
${ }^{30}$ Formally, for any $E S$ and any $c \in \mathbb{R}_{+}^{n}, \quad$ l.v. $(c ; p, w) \equiv$ $\min \left\{w \alpha_{l} \mid \alpha \in P^{\pi}(p, w) \& \widehat{\alpha} \geqq c\right\}$.

${ }^{31}$ See Yoshihara and Veneziani [53] for a detailed discussion.

${ }^{32}$ If $p \widehat{\alpha}^{p, w}=0$, we set $\tau^{c}=0$ by definition.
} 
income, and so $\tau^{c^{\nu}} w \alpha_{l}^{p, w}$ is the share of (the value of) social labour that $\nu$ receives by earning the income necessary to buy $c^{\nu}$. This is equivalent to "the amount of average social labor workers receive a claim to in the wage for each hour they actually work - that is, ... the average wage multiplied by the value of money" (Foley [11], p.43), or the value of labour power, according to the New Interpretation. Then, "Exploitation through the wage labor relation occurs when a worker expends more labor hours than he or she receives an equivalent for in wages" (Foley [11], p.122).

As in Roemer's [37] approach (and unlike in Morishima's), exploitation status in Definition 3 can be identified only if goods' prices are known, but social relations play a more central role, because the definition of exploitation requires knowledge of the social reproduction point, and it is related to the production and distribution of national income and social labour. Unlike Definitions 1 and 2, Definition 3 depends exclusively on empirically observable magnitudes. Nonetheless, the New Interpretation has been criticised because, unlike Definitions 1 and 2, the agents' actual consumption choices are only indirectly relevant to determine exploitation status, and unlike Definition 1, exploitation status depends on information about commodity prices.

This brief (and admittedly partial) survey shows that there are many possible approaches to explain what exploitation is and how it should be captured. The question then is how to adjudicate between them. Some preliminary answers are provided in the next section.

\section{Axiomatising Marxian exploitation}

In this section, we discuss two axioms incorporating some key properties that a definition of exploitation, and measure of exploitative relations, should satisfy and analyse their implications.

For any $E S$, let $W_{+} \equiv\left\{\nu \in \mathcal{N} \mid \omega^{\nu}=(0,0, \ldots, 0)^{\prime} \& w \lambda^{\nu}>0\right\}: W_{+}$is the set of agents with no initial endowments who supply some (productive) labour. Our axiomatic analysis focuses on the exploitation status of agents in $W_{+}$. Theoretically, this set is of focal interest from a Marxian perspective even in societies with a complex class structure: if any agents are exploited, propertyless agents who supply wage labour should be among them. Formally, as argued below, focusing on a strict subset of the set of agents makes the axiomatic restrictions weak and undemanding.

The first axiom is a domain condition capturing some minimal intuitions 
that represent the core of Marxian exploitation theory and that are shared by all of the main approaches.

Labour Exploitation with Heterogeneous Labour (LEH): Consider any ES. Given any definition of exploitation, there exists a profile of nonnegative vectors $\left(c_{e}^{1}, \ldots, c_{e}^{\left|W_{+}\right|}\right)$such that, for any $\nu \in W_{+}, p c_{e}^{\nu}=w \lambda^{\nu}$, and for some $\alpha^{c_{e}^{\nu}} \in \partial P$ with $\widehat{\alpha}^{c_{e}^{\nu}} \geqq c_{e}^{\nu}$ and $\widehat{\alpha}^{\nu_{e}^{\nu}} \ngtr c_{e}^{\nu}$ :

$$
\nu \text { is exploited if and only if } w \alpha_{l}^{c_{e}^{\nu}}<w \lambda^{\nu} \text {. }
$$

In order to interpret $\mathbf{L E H}$, recall that the exploitation status of agent $\nu$ is determined by the difference between the amount of labour 'contributed' and 'received' by $\nu$. As argued in section 3, in the main approaches consistent with the classical economists' view, the former quantity is given by the value of the labour supplied by $\nu, w \lambda^{\nu}$. But there are many possible views concerning the latter quantity. As a domain condition, LEH provides some minimal, key restrictions on the definition of the amount of labour that a theoretically relevant subset of agents receives. ${ }^{33}$

To be specific, LEH requires that the exploitation status of each propertyless worker $\nu \in W_{+}$be determined by identifying a nonnegative vector $c_{e}^{\nu}$ - call it an exploitation reference bundle (hereafter, ERB) - whose labour content - the amount of labour that $\nu$ receives - is the value of the labour necessary to produce the ERB as net output, $w \alpha_{l}^{c_{e}^{\nu}}$. If $\nu$ supplies $w \lambda^{\nu}$, and $w \lambda^{\nu}$ is more than $w \alpha_{l}^{c_{e}^{\nu}}$, then $\nu$ is regarded as contributing more labour than $\nu$ receives, and is thereby exploited.

The ERB must have two properties. First, it must be (just) affordable, at prices $p$, by a propertyless worker $\nu \in W_{+}$, who supplies $\lambda^{\nu}$ units of labour at wages $w: p c_{e}^{\nu}=w \lambda^{\nu}$. This embodies the idea that the amount of labour that $\nu \in W_{+}$receives depends on her income, or more precisely, it is determined by some reference bundle that $\nu$ can purchase. In standard approaches, the ERB is the bundle actually chosen by the agent. LEH is weaker in that it only requires that the ERB be potentially chosen.

Second, the ERB must be technically feasible with an efficient production process: $\alpha^{c_{e}^{\nu}} \in \partial P$ with $\widehat{\alpha}^{c_{e}^{\nu}} \geqq c_{e}^{\nu}$. This embodies the intuition that the amount of labour received by an agent is related to production conditions.

\footnotetext{
${ }^{33} \mathbf{L E H}$ only applies to labour-based definitions of exploitation. It is not relevant, for example, for Roemer's [37] property-relations definition. Related axioms are analysed in Yoshihara and Veneziani [52]; Yoshihara [51]; Veneziani and Yoshihara [49].
} 
More precisely, LEH states that the ERB be technologically feasible as net output, and its labour content is the amount of labour socially necessary to produce it. Observe that the axiom requires that the amount of labour associated with each ERB be uniquely determined with reference to production conditions, but it does not specify how such amount should be chosen. There may be in principle many (efficient) ways of producing the ERB, $c_{e}^{\nu}$, and thus of determining its labour content $w \alpha_{l}^{c_{e}^{\nu}} .34$

LEH incorporates the view that the sphere of production is central in determining exploitation status. For technology and production conditions determine the amount of labour $\alpha_{l}^{c_{e}^{\nu}}$ socially necessary to produce $c_{e}^{\nu}$, and technical innovations increasing labour productivity, and reducing $\alpha_{l}^{c_{e}^{\nu}}$, tend to increase exploitation. But LEH does not stipulate the primacy of the sphere of production, and it is consistent with approaches that allow for distribution and circulation to affect exploitation status. For prices and wages explicitly appear in the axiom, which allows one to capture the effect of changes in market conditions on exploitation. Thus, for example, the condition $p c_{e}^{\nu}=w \lambda^{\nu}$ is consistent with an emphasis on workers' purchasing power (and thus on workers qua consumers) such that a generalised decrease in wages, or increase in prices, would tend to increase exploitation for all $\nu \in W_{+}-$e.g., via a decrease in $\alpha_{l}^{c_{e}^{\nu}}$ associated with a decrease in $c_{e}^{\nu}$ to maintain $p c_{e}^{\nu}=w \lambda^{\nu}$. Given the focus on individual agents, however, LEH can also capture the (possibly divergent) effects of changes in relative prices (and wages) on the exploitation status of different groups of workers (and consumers) via changes in $c_{e}^{\nu}$, and the associated $\alpha_{l}^{c_{e}^{\nu}} .35$

LEH imposes weak and theoretically reasonable restrictions on the appropriate definition of exploitation, and all of the main approaches, including Definitions 1-3, satisfy it. ${ }^{36}$ LEH does not provide comprehensive conditions for the determination of exploitation status: it only focuses on the strict subset of agents who own no physical assets and supply some labour in return for

\footnotetext{
${ }^{34}$ Observe that LEH allows the ERB to be variable and a function of $(p, w)$. Further, once $c_{e}^{\nu}$ is identified, the existence of $\alpha^{c_{e}^{\nu}}$ is guaranteed by assumptions A0 and A2 in Appendix A.1.

${ }^{35}$ The role of exchange processes has been emphasised, for example, by Sweezy [44] and Baran and Sweezy [1] in their classic analysis of monopoly capitalism and of the effects of noncompetitive practices on prices, wages and the production and distribution of surplus value. We thank the Editors of this journal for this suggestion.

${ }^{36}$ For a proof of this claim, see the Addendum. It can also be proved that the definition of exploitation proposed in Flaschel [8] satisfies LEH.
} 
a wage and imposes no restrictions on the set of exploiters. More generally, LEH does not incorporate any assumptions on individual behaviour or on the structure of economic interactions. Nor is it based upon any (classical or neoclassical) equilibrium notion. It does not capture any causal mechanisms and embodies no assumptions on the nature of individuals or of social reality. It captures the properties of the concept of exploitation in a purely a posteriori - rather than predictionist - perspective, by focusing on the data emerging from economic processes at a given point in time.

Because LEH is a domain condition that captures some aspects of Marxian exploitation theory shared by all of the main approaches, further restrictions must be imposed in order to discriminate among alternative definitions. A key tenet of Marxian theory is the idea that in capitalist economies profits are closely related to the existence of exploitation. Given private ownership of productive assets, profits should be a counterpart of the transfer of social surplus and social labour from asset-poor agents to wealthy ones, and a general correspondence should exist between positive profits and the exploitation of at least the poorest segments of the working class. This is formalised in the next axiom.

Profit-Exploitation Correspondence Principle (PECP): Given any $E S$ such that $W_{+} \neq \varnothing$,

$$
\left[p \widehat{\alpha}^{p, w}-w \alpha_{l}^{p, w}>0 \text { if and only if every agent in } W_{+} \text {is exploited }\right] .
$$

In other words, at any $E S$, aggregate profits are strictly positive if and only if propertyless workers are exploited. This incorporates a key intuition of Marxian exploitation theory at the centre of the debates on the Fundamental Marxian Theorem discussed in the Introduction. Yet, PECP is both conceptually and formally distinct from the FMT. Conceptually, the FMT is conceived of as a causal statement: it is meant to prove that profits emerge from (are caused by) the exploitation of workers. Thus in the standard literature it is a result that may or may not hold in certain economies under a given definition of exploitation. PECP is instead conceived of as a fundamental, definitional property of Marxian exploitation theory. It is a statement about what exploitation is, or what intuitions it incorporates, and so it is formulated without specifying any definition: the appropriate 
definition should be such that propertyless workers are exploited if and only if profits are positive. ${ }^{37}$

Formally, unlike the FMT, PECP establishes a connection between the existence of aggregate profits and the exploitation status of a subset of the set of agents, rather than the aggregate rate of exploitation. Thus, PECP allows for the possibility that propertyless workers are a strict subset of the set of exploited agents, and when profits are zero it does not require that there be no exploitation in the economy, but only that some propertyless workers are not exploited. Moreover, like LEH, PECP focuses only on propertyless agents who supply some wage labour and so it imposes no constraints on the definition of exploitation whenever all agents possess some wealth, or propertyless agents are all unemployed $\left(W_{+}=\varnothing\right)$. This restriction is theoretically appropriate, because the exploitation status of agents who do not engage in any economic activities is unclear.

PECP establishes a rather weak link between exploitation and profits. ${ }^{38}$ It is therefore surprising that, in conjunction with LEH, it characterises a family of definitions of exploitation. ${ }^{39}$

Theorem 1: For any definition of exploitation satisfying $\mathbf{L E H}$, the following two statements are equivalent at any ES:

(1) PECP holds under this definition;

(2) if $\pi^{\max }>0$, then for all $\nu \in W_{+}$with $w \alpha_{l}^{c_{e}^{\nu}}>0$, there is $\alpha_{\pi}^{\nu} \in \partial P$ such that $\widehat{\alpha}_{\pi}^{\nu} \in \mathbb{R}_{+}^{n}, p \widehat{\alpha}_{\pi}^{\nu}>w \alpha_{\pi l}^{\nu}=w \lambda^{\nu}$ and $\left(\alpha_{\pi l}^{\nu}, \underline{\alpha}_{\pi}^{\nu}, \bar{\alpha}_{\pi}^{\nu}\right) \geqq \eta^{\nu}\left(\alpha_{l}^{c_{e}^{\nu}}, \underline{\alpha}^{c_{e}^{\nu}}, \bar{\alpha}^{c_{e}^{\nu}}\right)$ for some $\eta^{\nu}>1$.

Theorem 1 is mainly a technical result: it provides a condition that can be used to check whether a given definition satisfies PECP. As such, it does not identify a unique definition of exploitation that meets PECP, but rather a class of definitions satisfying condition (2). Yet it has relevant implications for the main received approaches. For there are economies in which condition (2) never holds, if Definitions 1 and 2 are adopted. In contrast, Definition 3 satisfies condition (2), and thus the PECP, in general. ${ }^{40}$

\footnotetext{
${ }^{37}$ Observe that PECP does not stipulate the primacy of the sphere of production and it is in principle consistent with theories of exploitation allowing for exploitative relations to be influenced at the point and in the process of exchange.

${ }^{38}$ Oberve also that PECP is silent on the set of exploiters.

${ }^{39}$ The proofs of all formal results are in Appendix A.2.

${ }^{40}$ It can also be proved that if a definition of exploitation satisfies LEH and PECP, then no agent in $W_{+}$is exploited whenever profits are zero.
} 
Corollary 1: There exist ES's such that neither Definition 1 nor Definition 2 satisfies PECP. Instead, Definition 3 satisfies PECP at any ES.

Theorem 1 and Corollary 1 show that, contrary to a widespread belief, general technologies with heterogeneous labour do not necessarily pose insurmountable problems for Marxian exploitation theory. They prove the existence of a non-empty class of definitions that preserve a robust relation between exploitation and profits in general economies with heterogeneous labour.

Corollary 1 also provides support to the New Interpretation, as the only definition, among the main ones in the literature, that satisfies PECP in general. It is worth emphasising, however, that Definitions 1 and 2 fail to satisfy PECP because of some deep formal and conceptual issues that are unrelated to the treatment of heterogeneous labour. To see this, consider an economic environment with two commodities $(n=2)$, one type of homogeneous labour $(\mathcal{T}=\{1\}), N_{c}>0$ capitalists who own some physical endowments and $N_{w}>0$ propertyless agents, with $N=N_{c}+N_{w}$. Suppose that there is only one production process where both commodities are jointly produced with fixed input-output proportions and homogeneous labour, such that the production set is $P=\left\{\alpha=k \alpha^{0} \mid k \geq 0\right\}$, where $\alpha^{0} \equiv(-1,-(1,0),(3,1))$. Then it is easy to show that there exist $E S$ 's with $p_{1}>0$ and $p_{2}>0$, $p_{1}+p_{2}=1$, and $w=1$ in which each propertyless agent $\nu$ supplies one unit of (homogeneous) labour and purchases a bundle $c^{\nu}=(1,1)$, while capitalists do not work at all. At these $E S$ 's, aggregate profits are $p \widehat{\alpha}^{p, w}-w \alpha_{l}^{p, w}>0$ where $\alpha^{p, w}=N_{w} \alpha^{0}$ is the unique profit rate maximiser. Nonetheless, according to both Definition 1 and Definition 2, the labour received by each propertyless worker $\nu$ is equal to one - l.v. $\left(c^{\nu} ; w\right)=l . v \cdot\left(c^{\nu} ; p, w\right)=1-$ and therefore none of them is exploited. ${ }^{41}$

This example shows that the inability of Definitions 1 and 2 to capture the relation between exploitation and profits does not derive from the presence of heterogeneous labour. Rather, the problem lies in their notion of labour received by agents in economies with general technologies allowing for joint products, fixed capital, choice of techniques, and so on. Indeed, it is possible to prove that in production economies with only circulating capital and a single production technique available to produce each commodity with fixed coefficients, a large subset of the set of definitions of exploitation satis-

\footnotetext{
${ }^{41}$ In contrast, l.v. $\left(c^{\nu} ; p, w, \alpha^{p, w}\right)=\frac{1}{1+p_{1}}<1$ and all propertyless workers are exploited according to Definition 3.
} 
fying LEH (including all of Definitions 1-3) preserves the relation between exploitation and profits. Formally:

Proposition 1: Suppose that $P$ is representable by a Leontief production technique $(\boldsymbol{A}, \boldsymbol{L})$. Then at any ES, PECP holds under any definition of exploitation satisfying $\mathbf{L E H}$ with $\widehat{\alpha}^{c_{e}^{\nu}} \nsucceq c_{e}^{\nu}$.

\section{Conclusions}

This paper explores a novel axiomatic approach to Marxian exploitation theory. Two properties - a domain axiom and the Profit-Exploitation Correspondence Principle - are analysed, which incorporate some widely shared intuitions concerning the normative and positive foundations of the concept of exploitation. Contrary to the received view, a nonempty class of definitions of exploitation is characterised, which preserve the relation between profits and the exploitation of propertyless workers in general economies with a complex class structure, heterogeneous agents, complex technologies with heterogeneous labour inputs, general market structures, and so on. Interestingly, however, among the main approaches, only the 'New Interpretation' is shown to satisfy PECP in general. Given the theoretical relevance of PECP in Marxian theory, this provides strong support for Definition 3 above.

To be sure, the relation between exploitation and profits is only one albeit important - aspect of Marxian theory and the results in this paper do not exhaust the analysis of the concept of exploitation. Yet, they do show the potential of the axiomatic method and in closing the paper we briefly mention some lines for further research.

First of all, the concept of exploitation is meant to be a diagnostic of the characteristics of the social structure, both in its power dimension and in its inequality dimension. In this paper, we have focused on the latter. It would be important to extend our analysis to incorporate power, and coercive social relations into the axiomatic framework.

Further, Theorem 1 does not identify a single definition that meets PECP, but rather a class of definitions. It would be interesting to analyse whether a unique definition can be characterised by imposing further properties, and if so, whether such definition is indeed the 'New Interpretation'.

This is an open question, but two points are worth making that suggest that the key insights of the paper are indeed robust. First, in the standard 
Okishio-Morishima approach, the existence of exploitation is just a numerical representation of the existence of surplus products. Thus, the FMT establishes the equivalence between positive profits and the productiveness of the economy measured in terms of the labour numéraire. Yet, it has been proved that a similar result holds when productiveness is measured in terms of any other good, thus raising doubts on the significance of the relation between exploitation and profits (Roemer [37]). Yoshihara and Veneziani [54] have proved that this is not true if the 'New Interpretation' is adopted: no equivalence between profits and exploitation holds if another commodity is used to define exploitation.

Second, in Marxian theory, the social positions identified by the notion of exploitation are internally related: 'they are what they are ... by virtue of the relation to other in which they stand' (Lawson [22], p. 17). The existence of an exploiter is inextricably linked to the existence of exploited agents and 'you cannot have the one without the other' (ibid.). Perhaps surprisingly, Yoshihara and Veneziani [52] have proved that the 'New Interpretation' is the only conceivable definition that possesses this relational property.

In summary, this paper provides a general theoretical framework to analyse the notion of exploitation. The results derived shed light on some important (and vexed) issues. More importantly, they show that it is fruitful to take the axiomatic road to exploitation theory, even though there are still a million miles to go.

\section{A Formal analysis}

\section{A.1 Assumption on technology}

Let $\mathbf{0}$ be the null vector. The following assumptions on $P$ hold throughout the paper.

Assumption 0 (A0). $P$ is a closed convex cone in $\mathbb{R}^{2 n+T}$ and $\mathbf{0} \in P$.

Assumption 1 (A1). For all $\alpha \in P$, if $\bar{\alpha} \geq \mathbf{0}$ then $\alpha_{l} \geq \mathbf{0}$ and $\underline{\alpha} \geq \mathbf{0}$.

Assumption 2 (A2). For all $c \in \mathbb{R}_{+}^{n}$, there is a $\alpha \in P$ such that $\widehat{\alpha} \geqq c$. 


\section{A.2 Proofs}

Proof of Theorem 1: Consider any ES. If $W_{+}=\varnothing$, the equivalence is immediately established, for both PECP and condition (2) are vacuously satisfied. Therefore, in the rest of the proof, suppose that $W_{+} \neq \varnothing$. Let $N^{\text {ted }} \subseteq \mathcal{N}$ denote the set of exploited agents.

$(2) \Rightarrow(1)$ : Suppose that if $\pi^{\max }>0$, then for each $\nu \in W_{+}$with $w \alpha_{l}^{c_{e}^{\nu}}>0$, there exists $\alpha_{\pi}^{\nu} \in \partial P$ such that $p \widehat{\alpha}_{\pi}^{\nu}>w \alpha_{\pi l}^{\nu}=w \lambda^{\nu}$ and $\left(\alpha_{\pi l}^{\nu}, \underline{\alpha}_{\pi}^{\nu}, \bar{\alpha}_{\pi}^{\nu}\right) \geqq$ $\eta^{\nu}\left(\alpha_{l}^{c_{e}^{\nu}}, \underline{\alpha}^{c_{e}^{\nu}}, \bar{\alpha}^{c_{e}^{\nu}}\right)$ for some $\eta^{\nu}>1$.

Let $p \widehat{\alpha}^{p, w}-w \alpha_{l}^{p, w}=0$. Then by the definition of $E S, \pi^{\max }=0$ and condition (2) is vacuously satisfied. By LEH, for each $\nu \in W_{+}, c_{e}^{\nu} \in \mathbb{R}_{+}^{n}$, $p c_{e}^{\nu}=w \lambda^{\nu}>0$ and $\alpha^{c_{e}^{\nu}} \in \partial P$ with $\widehat{\alpha}^{c_{e}^{\nu}} \geqq c_{e}^{\nu}$. Therefore, noting that $p \widehat{\alpha}^{c_{e}^{\nu}} \geqq p c_{e}^{\nu}=w \lambda^{\nu}>0, \pi^{\max }=0$ implies that $w \alpha_{l}^{c_{e}^{\nu}} \geqq w \lambda^{\nu}$. Hence, by LEH, $\nu \notin N^{\text {ted }}$ holds for all $\nu \in W_{+}$.

Let $p \widehat{\alpha}^{p, w}-w \alpha_{l}^{p, w}>0$ so that $\pi^{\max }>0$. Consider any $\nu \in W_{+}$. If $w \alpha_{l}^{c_{e}^{\nu}}=0$, then by definition $w \alpha_{l}^{c_{e}^{\nu}}<w \lambda^{\nu}$. If $w \alpha_{l}^{c_{e}^{\nu}}>0$, then by condition (2), $\alpha_{\pi}^{\nu} \in \partial P$ with $w \alpha_{\pi l}^{\nu}=w \lambda^{\nu}>0$ and $\eta^{\nu}>1$ together imply $w \alpha_{l}^{c_{e}^{\nu}}<w \lambda^{\nu}$. Thus, by LEH, $\nu \in N^{\text {ted }}$ holds for any $\nu \in W_{+}$.

In summary, (2) implies that PECP holds under any definition of exploitation satisfying LEH.

$(1) \Rightarrow(2)$ : Suppose that $p \widehat{\alpha}^{p, w}-w \alpha_{l}^{p, w}>0 \Leftrightarrow N^{\text {ted }} \supseteq W_{+}$.

Suppose that $\pi^{\max }>0$. By the definition of $E S, p \widehat{\alpha}^{p, w}-w \alpha_{l}^{p, w}>0$ holds, and by LEH and PECP, for each $\nu \in W_{+}$, there exist $c_{e}^{\nu} \in \mathbb{R}_{+}^{n}$ and $\alpha^{c_{e}^{\nu}} \in \partial P$ with $\widehat{\alpha}^{c_{e}^{\nu}} \geqq c_{e}^{\nu}$ and $\widehat{\alpha}^{c_{e}^{\nu}} \ngtr c_{e}^{\nu}$ such that $p c_{e}^{\nu}=w \lambda^{\nu}>0$ and $w \alpha_{l}^{c_{e}^{\nu}}<w \lambda^{\nu}$. Consider $\nu \in W_{+}$such that $w \alpha_{l}^{c_{e}^{\nu}}>0$. Then let $\eta^{\nu} \in$ $\mathbb{R}_{+}$be such that $\eta^{\nu} w \alpha_{l}^{c_{e}^{\nu}}=w \lambda^{\nu}$ and let $\alpha_{\pi}^{\nu} \equiv \eta^{\nu} \alpha^{c_{e}^{\nu}}$. Since $\alpha^{c_{e}^{\nu}} \in \partial P$ with $\widehat{\alpha}^{c_{e}^{\nu}} \geqq c_{e}^{\nu} \geqq \mathbf{0}$, then $\widehat{\alpha}_{\pi}^{\nu} \in \mathbb{R}_{+}^{n}$ and, by A0, $\alpha_{\pi}^{\nu} \in \partial P$. Moreover, by construction, $\left(\alpha_{\pi l}^{\nu}, \underline{\alpha}_{\pi}^{\nu}, \bar{\alpha}_{\pi}^{\nu}\right) \geqq \eta^{\nu}\left(\alpha_{l}^{c_{e}^{\nu}}, \underline{\alpha}^{c_{e}^{\nu}}, \bar{\alpha}^{c_{e}^{\nu}}\right)$ for some $\eta^{\nu}>1$. Finally, $p \widehat{\alpha}_{\pi}^{\nu} \geqq \eta^{\nu} p \widehat{\alpha}_{e}^{c_{e}^{\nu}}>p \widehat{\alpha}^{c_{e}^{\nu}} \geqq p c_{e}^{\nu}=w \lambda^{\nu}=w \alpha_{\pi l}^{\nu}$ holds.

In summary, if PECP holds, then (2) holds under any definition of exploitation satisfying $\mathbf{L E H}$.

Proof of Corollary 1: For a proof that Definitions 1 and 2 do not satisfy PECP, see Veneziani and Yoshihara [49], which proves the result in a subset of the economic environments considered here.

We prove that Definition 3 satisfies condition (2) of Theorem 1. Consider any $E S$ with $W_{+} \neq \varnothing$. 
Suppose $\pi^{\max }>0$. By the definition of $E S$, this implies $p \widehat{\alpha}^{p, w}-w \alpha_{l}^{p, w}>0$; and since $w \lambda^{\nu}>0$ for all $\nu \in W_{+}$then $w \alpha_{l}^{p, w} \geqq \sum_{\nu \in W_{+}} w \lambda^{\nu}>0$. Then, for all $\nu \in W_{+}$, let $\alpha_{\pi}^{\nu}=\frac{w \lambda^{\nu}}{w \alpha_{l}^{p, w}} \alpha^{p, w}: \alpha^{p, w} \in \partial P$ holds by condition (iii) of $E S$ and

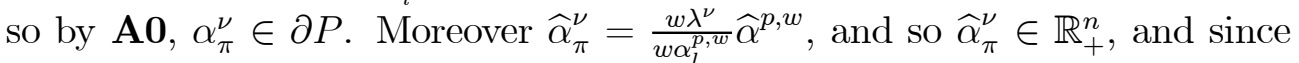
$p \widehat{\alpha}^{p, w}-w \alpha_{l}^{p, w}>0$, it follows that $p \widehat{\alpha}_{\pi}^{\nu}>w \alpha_{\pi l}^{\nu}=w \lambda^{\nu}$.

Finally, under Definition 3, $\alpha^{c_{e}^{\nu}}=\tau^{c^{\nu}} \alpha^{p, w}$ holds, where $\tau^{c^{\nu}}=\frac{p c^{\nu}}{p \widehat{\alpha}^{p, w}}$ for all $\nu \in W_{+}$. Hence, $\left(\alpha_{\pi l}^{\nu}, \underline{\alpha}_{\pi}^{\nu}, \bar{\alpha}_{\pi}^{\nu}\right) \geqq \eta^{\nu}\left(\alpha_{l}^{c_{e}^{\nu}}, \underline{\alpha}^{c_{e}^{\nu}}, \bar{\alpha}^{c_{e}^{\nu}}\right)$ for some $\eta^{\nu}>1$ if and only if $\frac{w \lambda^{\nu}}{w \alpha_{l}^{p, w}}\left(\alpha_{l}^{p, w}, \underline{\alpha}^{p . w}, \bar{\alpha}^{p, w}\right) \geqq \eta^{\nu} \frac{p c^{\nu}}{p \widehat{\alpha}^{p, w}}\left(\alpha_{l}^{p, w}, \underline{\alpha}^{p . w}, \bar{\alpha}^{p, w}\right)$ for some $\eta^{\nu}>1$, and the latter inequality holds for all $\nu \in W_{+}$whenever $p \widehat{\alpha}^{p, w}-w \alpha_{l}^{p, w}>0$, since $p c^{\nu}=w \lambda^{\nu}$ for all $\nu \in W_{+}$by condition (iv) of $E S$.

In summary, condition (2) of Theorem 1 holds at any ES.

Proof of Proposition 1: Suppose $P$ is representable by a Leontief production technique $(\boldsymbol{A}, \boldsymbol{L})$. Consider any $E S$. If $\sum_{\nu \in \mathcal{N}} c^{\nu}=\mathbf{0}$, then condition (iv) of $E S$ implies $W_{+}=\varnothing$, and so PECP is vacuously satisfied. Therefore, in what follows, assume $\sum_{\nu \in \mathcal{N}} c^{\nu} \geq \mathbf{0}$.

By LEH, for any $\nu \in W_{+}$, there exists $c_{e}^{\nu} \geq \mathbf{0}$ such that $p c_{e}^{\nu}=w \lambda^{\nu}$ and

$$
\alpha^{c_{e}^{\nu}}=\left(-\boldsymbol{v} c_{e}^{\nu},-\boldsymbol{A}(\boldsymbol{I}-\boldsymbol{A})^{-1} c_{e}^{\nu},\left[\boldsymbol{I}+\boldsymbol{A}(\boldsymbol{I}-\boldsymbol{A})^{-1}\right] c_{e}^{\nu}\right)
$$

where $\boldsymbol{v} \equiv \boldsymbol{L}(\boldsymbol{I}-\boldsymbol{A})^{-1}>\mathbf{0}$ by the indecomposability of $\boldsymbol{A}$. By $\mathbf{L E H}, \nu$ is exploited if and only if $w \alpha_{l}^{c_{e}^{\nu}}=w \boldsymbol{v} c_{e}^{\nu}<w \lambda^{\nu}$.

Suppose $\pi^{\max }>0$. Then at any $E S$, it must be $p>p \boldsymbol{A}+w \boldsymbol{L}$. (To see this, let $x$ be the aggregate activity level at the $E S$. If $p_{j} \leqq p \boldsymbol{A}_{j}+w \boldsymbol{L}_{j}$, for some $j$, then $x_{j}=0$ must hold by part (ii) of the definition of $E S$. However, by part (i) it must be $(\boldsymbol{I}-\boldsymbol{A}) x \geqq \sum_{\nu \in \mathcal{N}} c^{\nu}$ and since $\sum_{\nu \in \mathcal{N}} c^{\nu} \geq$ 0, the indecomposability of $\boldsymbol{A}$ implies $x \geqq(\boldsymbol{I}-\boldsymbol{A})^{-1}\left(\sum_{\nu \in \mathcal{N}} c^{\nu}\right)>\mathbf{0}$, a contradiction.) Then, $p>w \boldsymbol{v}$ holds by $(\boldsymbol{I}-\boldsymbol{A})^{-1}>\mathbf{0}$. Therefore by $\mathbf{L E H}$, for any $\nu \in W_{+}$, it follows that $w \lambda^{\nu}=p c_{e}^{\nu}>w \boldsymbol{v} c_{e}^{\nu}=w \alpha_{l}^{c_{e}^{\nu}}$ and $\nu$ is exploited.

Suppose $\pi^{\max }=0$. Then at any $E S, p \leqq p \boldsymbol{A}+w \boldsymbol{L}$ and $p \leqq w \boldsymbol{v}$. Hence, by $\mathbf{L E H}$, for any $\nu \in W_{+}, w \lambda^{\nu}=p c_{e}^{\nu} \leqq w \boldsymbol{v} c_{e}^{\nu}=w \alpha_{l}^{c_{e}^{\nu}}$, and $\nu$ is not exploited.

\section{References}

[1] Baran, P.A., Sweezy, P., 1966. Monopoly capital. Monthly Review Press, New York. 
[2] Bowles, S., Gintis, H., 1977. The Marxian Theory of Value and Heterogeneous Labour: A Critique and Reformulation. Cambridge Journal of Economics 1, 173-192.

[3] Downward, P., (ed.), 2003. Applied Economics and the Critical Realist Critique. Routledge, London.

[4] Downward, P., Finch, J.H., Ramsay, J., 2003. Seeking a role for empirical analysis in critical realist explanation. In: Downward, P., (ed.). Applied Economics and the Critical Realist Critique. Routledge, London.

[5] Duménil, G., 1980. De la Valeur aux Prix de Production. Economica, Paris.

[6] Duménil, G., Foley, D.K., Lévy, D., 2009. A Note on the Formal Treatment of Exploitation in a Model with Heterogeneous Labor. Metroeconomica $60,560-567$.

[7] Fine, B., 2010. Locating Financialisation. Historical Materialism 18, 97116.

[8] Flaschel, P., 2010. Topics in Classical Micro- and Macroeconomics. Springer, New York.

[9] Flaschel, P., Franke, R., Veneziani, R., 2013. Labor Productivity and the Law of Decreasing Labor Content. Cambridge Journal of Economics $37,379-402$.

[10] Foley, D.K., 1982. The Value of Money, the Value of labour Power, and the Marxian Transformation Problem. Review of Radical Political Economics 14, 37-47.

[11] Foley, D.K., 1986. Understanding Capital. Harvard University Press, Cambridge, MA.

[12] Foster, J.E., 1994. Normative Measurement: Is Theory Relevant? American Economic Review 84, 365-370.

[13] Freeman, A., Carchedi, G., (eds) 1996. Marx and Non-equilibrium Economics. Edward Elgar, Aldershot. 
[14] Fujimori, Y., 1982. Modern Analysis of Value Theory. Springer-Verlag, Berlin.

[15] Katzner, D.W., 2009. Analysis without Measurement. Cambridge University Press, Cambridge.

[16] Krause, U., 1982. Heterogeneous Labour and the Fundamental Marxian Theorem. Review of Economic Studies 48, 173-178.

[17] Kurz, H.D., Salvadori, N., 1995. Theory of Production: A long-Period Analysis. Cambridge University Press, Cambridge.

[18] Lapavitsas, C., 2009. Financialised Capitalism: Crisis and Financial Expropriation. Historical Materialism 17, 114-148

[19] Lapavitsas, C., 2013. Profiting without producing. Verso, London.

[20] Lawson, T., 1997. Economics and Reality. Routledge, London.

[21] Lawson, T., 1999. Connections and Distinctions: Post Keynesianism and Critical Realism. Journal of Post-Keynesian Economics 22, 3-14.

[22] Lawson, T., 2003. Reorienting Economics. Routledge, London.

[23] Lawson, T., 2009. The current economic crisis: its nature and the course of academic economics. Cambridge Journal of Economics 33, 759-777.

[24] Lawson, T., 2013. What is this 'school' called neoclassical economics? Cambridge Journal of Economics 37, 947-983.

[25] Martins, N.O., 2006. Capabilities as causal powers. Cambridge Journal of Economics 30, 671-685.

[26] Martins, N.O., 2012. Sen, Sraffa and the revival of classical political economy. Journal of Economic Methodology 19, 143-157.

[27] Marx, K., 1954 [1867]. Capital, Vol.I. London, Lawrence \& Wishart.

[28] Marx, K., 1959 [1894]. Capital, Vol.III. London, Lawrence \& Wishart.

[29] Mohun, S., 2003. On the TSSI and the Exploitation Theory of Profit. Capital and Class 81, 85-102. 
[30] Morishima, M., 1973. Marx's Economics. Cambridge University Press, Cambridge.

[31] Morishima, M., 1974. Marx in the Light of Modern Economic Theory. Econometrica 42, 611-632.

[32] Okishio, N., 1957. Theory of Reproduction (in Japanese). Sobun Publisher.

[33] Okishio, N., 1963. A mathematical note on Marxian Theorems. Weltwirtschaftliches Archiv 91, 287-299.

[34] Okishio, N., 1965. Fundamental Theory of Capitalist Economy (in Japanese). Sobun Publisher.

[35] Ricardo, D., 2004 [1819]. The Principles of Political Economy and Taxation. Dover Publication.

[36] Roemer, J.E., 1981. Analytical foundations of Marxian economic theory. Harvard University Press, Cambridge, MA.

[37] Roemer, J.E., 1982. A General Theory of Exploitation and Class. Harvard University Press, Cambridge, MA.

[38] Sen, A.K., 1978. On the labour theory of value: some methodological issues. Cambridge Journal of Economics 2, 175-190.

[39] Sen, A.K., 1985a. Commodities and Capabilities. North-Holland, Amsterdam.

[40] Sen, A.K., 1999. The Possibility of Social Choice. American Economic Review 89, 349-378.

[41] Setterfield, M., 2003. Critical realism and formal modelling: incompatible bedfellows? In: Downward, P., (ed.). Applied Economics and the Critical Realist Critique. Routledge, London.

[42] Smith, A., 2000 [1776]. The Wealth of Nations. Modern Library, New York.

[43] Steedman, I., 1977. Marx after Sraffa. New Left Books, London. 
[44] Sweezy, P., 1970 [1940]. The Theory of Capitalist Development. Monthly Review Press, New York.

[45] Thomson, W., 2001. On the axiomatic method and its recent applications to game theory and resource allocation. Social Choice and Welfare $18,327-386$.

[46] Veneziani, R., 2004. The Temporal Single-System Interpretation of Marx's Economics: A Critical Evaluation. Metroeconomica 55, 96-114.

[47] Veneziani, R., 2007. Exploitation and Time. Journal of Economic Theory $132,189-207$.

[48] Veneziani, R., 2013. Exploitation, Inequality, and Power. Journal of Theoretical Politics 25, 526-545.

[49] Veneziani, R., Yoshihara, N., 2015. Exploitation in economies with heterogeneous preferences, skills and assets: An axiomatic approach. Journal of Theoretical Politics 27, 8-33.

[50] Wright, E. O., 2000. Class, Exploitation, and Economic Rents: Reflections on Sorensen's "Sounder Basis". The American Journal of Sociology 105, 1559-71.

[51] Yoshihara, N., 2010. Class and Exploitation in General Convex Cone Economies. Journal of Economic Behavior $\&$ Organization 75, 281-296.

[52] Yoshihara, N., Veneziani, R., 2009. Exploitation as the Unequal Exchange of Labour: An Axiomatic Approach. WP N.655, Queen Mary University of London.

[53] Yoshihara, N., Veneziani, R., 2012. Profits and Exploitation: A Reappraisal. Advances in Mathematical Economics 16, 85-109.

[54] Yoshihara, N., Veneziani, R., 2013. Exploitation of Labour and Exploitation of Commodities: a 'New Interpretation'. Review of Radical Political Economics 45, 517-524.

[55] Yoshihara, N., Veneziani, R., 2014. The Measurement of Labour Content: A General Approach. DP 2013-5, University of Massachusetts, Amherst. 